\title{
Clinical significance of neutrophil to lymphocyte ratio and platelet to lymphocyte ratio in acute cerebral hemorrhage with gastrointestinal hemorrhage, and logistic regression analysis of risk factors
}

\author{
YU ZOU, WEI ZHANG, CHUANJUN HUANG and YANGQING ZHU \\ Department of Neurosurgery, The First People's Hospital of Wujiang District, Suzhou, Jiangsu 215200, P.R. China
}

Received September 22, 2018; Accepted June 6, 2019

DOI: $10.3892 /$ etm.2019.7778

\begin{abstract}
The aim of the present study was to determine the predictive value of the neutrophil to lymphocyte ratio (NLR) and platelet to lymphocyte ratio (PLR) in patients with acute cerebral hemorrhage with or without gastrointestinal hemorrhage. Risk factors of gastrointestinal hemorrhage in patients with acute cerebral hemorrhage were also assessed. A total of 335 patients with acute cerebral hemorrhage admitted to our hospital between January 2012 and January 2017 were enrolled. The 86 patients who experienced gastrointestinal hemorrhage during hospitalization were selected as the observation group, while the 249 remaining cases were assigned to the negative control group. The neutrophil, white blood cell and platelet count, as well as the NLR and PLR of each subject were recorded. Furthermore, sex, age, blood pressure, the site of cerebral hemorrhage, the amount of bleeding, the Glasgow Coma Scale (GCS) score and presence of hematosepsis were also recorded and assessed as potential risk factors for gastrointestinal hemorrhage in patients with acute cerebral hemorrhage. The NLR and PLR were markedly higher in the observation group compared with those in the negative control group. Furthermore, the NLR and PLR in the observation group were negatively associated with the 90-day overall survival of patients with acute cerebral hemorrhage and gastrointestinal hemorrhage. In the negative control group, only the PLR was negatively associated with overall survival. Logistic regression analysis indicated that a cerebral hemorrhage volume of $>30 \mathrm{ml}$, lower GCS score and hematosepsis were independently associated with gastrointestinal hemorrhage in patients with acute cerebral hemorrhage $(\mathrm{P}<0.05)$. A high NLR and
\end{abstract}

Correspondence to: Dr Yangqing Zhu, Department of Neurosurgery, The First People's Hospital of Wujiang District, 169 Park Road, Songling Town, Wujiang, Suzhou, Jiangsu 215200, P.R. China E-mail: godb93@163.com

Key words: acute cerebral hemorrhage, gastrointestinal hemorrhage, neutrophil to lymphocyte ratio, platelet to lymphocyte ratio
PLR indicated an elevated risk of gastrointestinal hemorrhage in patients with acute cerebral hemorrhage. A higher NLR and PLR were also negatively associated with overall survival and prognosis of patients with cerebral hemorrhage. In addition, a cerebral hemorrhage volume of $>30 \mathrm{ml}$, lower GCS score and hematosepsis were independent risk factors of gastrointestinal hemorrhage in patients with acute cerebral hemorrhage.

\section{Introduction}

Acute cerebral hemorrhage is a type of primary non-traumatic intracerebral hemorrhage, accounting for $15-20 \%$ of all strokes, and the mortality rate is as high as approximately $30 \%$. To date, no specific treatment method has been developed to effectively reduce mortality of acute cerebral hemorrhage (1). The morbidity of cerebral hemorrhage is twice that of ischemic cerebrovascular disease (2), and the 30-day mortality is as high as $41 \%$ (3). Studies have indicated that peripheral blood leukocytes are associated with the prognosis of patients with cerebral hemorrhage. Leukocytes have an important role in the secondary damage caused by inflammatory reactions after intracerebral hemorrhage (4), which are associated with early neurologic impairment in patients with cerebral hemorrhage (5). In a mouse model of intracerebral hemorrhage, it has been demonstrated that peripheral blood mononuclear cells significantly aggregate at the hemorrhage site compared with other types of inflammatory cell (6). Matrix metalloproteinases (MMPs) are considered as important inflammatory mediators (7), and neutrophils are the major source of MMP9 (8). Previous studies have indicated that peripheral blood leukocytes are associated with inflammatory reactions in acute cerebral hemorrhage. However, the mechanism of the inflammatory response after intracerebral hemorrhage has not been fully elucidated.

Gastrointestinal bleeding caused by Cushing ulcer is one of the complications of acute cerebral hemorrhage, with a morbidity of $48.3 \%$ and a mortality of as high as $87.9 \%$. Therefore, identifying and preventing gastrointestinal hemorrhage in patients with cerebral hemorrhage may effectively reduce the mortality rate. Previous studies have indicated that NLR and PLR are closely linked to the degree of severity and prognosis of inflammation-associated diseases, 
including malignant tumors and acute pancreatitis $(9,10)$. Based on clinical data and preliminary observations, our group determined that the neutrophil count (NEU) has a certain value in identifying patients with acute cerebral hemorrhage combined with gastrointestinal hemorrhage. The present study aimed to explore the clinical significance of NLR and PLR in the diagnosis and prognosis of acute cerebral hemorrhage.

\section{Patients and methods}

Subjects. A total of 335 patients with acute cerebral hemorrhage diagnosed by clinical examinations and cranial computed tomography (CT) at the First People's Hospital of Wujiang District (Suzhou, China) between January 2012 and January 2017 were selected. The cohort comprised 181 male patients and 154 female patients with an age range of 32-87 years (median age, 60 years). All enrolled patients were admitted due to acute cerebral hemorrhage. Among them, 86 patients who experienced gastrointestinal hemorrhage were selected as the observation group and the remaining 249 were selected as a negative control group. The diagnostic criteria for gastrointestinal hemorrhage were as follows: Bloody or brown vomitus or gastric tube drainage, or positive occult blood test; tarry stool or melena or positive fecal occult blood test. In all cases, gastrointestinal hemorrhage occurred within 2 weeks of acute cerebral hemorrhage. Exclusion criteria included admission at $24 \mathrm{~h}$ after the onset, history of blood transfusion within 3 months, comorbidity of hematological diseases, tumors, severe dysfunctions of liver and kidney, endocrine or digestive system diseases, and various autoimmune diseases, as well as taking of immunosuppressants, a history of acute infection or antibiotic medication in last 3 weeks, and a history of stroke. The present study was approved by the ethics committee of the First People's Hospital of Wujiang District (Suzhou, China). Written informed consent was obtained from all participants prior to the study.

Collection of basic characteristics. Peripheral blood samples of all subjects were collected within $24 \mathrm{~h}$ after admission and every 2 days since after. The NEU, white blood cell count (WBC), platelet count (PLT), NLR and PLR of each subject were recorded. Furthermore, sex, age, blood pressure, the site of cerebral hemorrhage and the volume of bleeding, the Glasgow Coma Scale (GCS) score and presence of hematosepsis were also recorded.

Statistical analysis. SPSS 22.0 statistical software (IBM Corp.) was used for data analysis. Measurement data were expressed as the mean \pm standard deviation. Student's t-test or the Chi-squared test were used to compare differences between the two groups. Kaplan-Meier analysis was performed to draw the survival curves and the log-rank test was utilized to assess differences in survival between the groups. Univariate Cox hazard analysis and stepwise multivariate Cox hazard analysis were performed to identify independent risk factors of acute cerebral hemorrhage combined with gastrointestinal hemorrhage. $\mathrm{P}<0.05$ was considered to indicate statistical significance.

\section{Results}

Basic characteristics of the subjects. A total of 355 patients with acute cerebral hemorrhage combined with gastrointestinal hemorrhage diagnosed by clinical examinations and cranial CT at our hospital between January 2012 and January 2017 were selected for inclusion. Based on the presence of the complication of gastrointestinal hemorrhage, 86 subjects were assigned to the observation group and the 249 remaining subjects were enrolled in the negative control group. Sex, age, blood pressure, the site of cerebral hemorrhage, the amount of bleeding, GCS score and presence of hematosepsis were recorded and compared between the two groups. The specific information of the subjects is listed in Table I. There was no significant difference in sex, age and blood pressure between the two groups, while the observation group had a higher GCS score, volume of cerebral hemorrhage and rate of hematosepsis.

Comparisons of NLR and PLR. Within 5 days of admission, the NLR and PLR were markedly higher in the observation group than those in the negative control group. In particular, there were significant differences in the NLR on the 1st and 3rd day after admission between the two groups (all $\mathrm{P}<0.05$ ). However, on the 5th day, no significant difference in NLR was observed. Furthermore, significant differences in the PLR on the 1st, 3rd and 5rd day after admission were identified between the two groups (all $\mathrm{P}<0.05$; Fig. 1).

Effects of NLR and PLR on overall survival. The NLR and PLR of each subject with acute cerebral hemorrhage were determined on the first day of admission and the influence of these ratios at baseline on the 90-day survival rate was assessed. The NLR and PLR in the observation group were $5.32 \pm 1.02$ and $151.20 \pm 6.35$, respectively, while they were $6.89 \pm 1.93$ and $192.76 \pm 8.26$ in the control group, respectively. Kaplan-Meier analysis was then performed to assess the effect of an NLR or PLR above/below the above-mentioned mean values used as a cutoff on overall survival in each of the two groups over 90 days (Fig. 2). The results indicated that the NLR and PLR in the observation group were negatively associated with the overall survival of patients with acute cerebral hemorrhage and the survival rates of the low NLR or PLR groups were significantly higher than that of the high NLR or PLR group $(\mathrm{P}<0.05$; Fig. $2 \mathrm{C}$ and $\mathrm{D})$. However, no significant association between NLR and overall survival was observed in the control group (Fig. 2A). The PLR in the control group was negatively associated with overall survival $(\mathrm{P}<0.05$; Fig. 2B).

Multivariate logistic regression analysis. To determine factors that are independent predictors of gastrointestinal hemorrhage in patients with acute cerebral hemorrhage, multivariate logistic regression analysis was performed. A blood pressure of $>180 / 100 \mathrm{mmHg}$, cerebral hemorrhage $>30 \mathrm{ml}$, lower GCS score, hematosepsis and the site of cerebral hemorrhage were considered as independent variables $(\mathrm{P}<0.05$; Table II), while gastrointestinal hemorrhage in patients with acute cerebral hemorrhage was taken as the dependent variable. The results suggested that cerebral hemorrhage $>30 \mathrm{ml}$, lower GCS score and hematosepsis were independent predictors of 
Table I. Baseline clinicopathological characteristics of the patients.

\begin{tabular}{lcc}
\hline Parameter & Observation group $(\mathrm{n}=86)$ & Control group $(\mathrm{n}=249)$ \\
\hline Sex & & $136(54.6)$ \\
Male & $45(52.3)$ & $113(45.4)$ \\
Female & $41(47.7)$ & $61.8 \pm 5.3$ \\
Age (years) & $59.3 \pm 6.2$ & \\
Blood pressure $(\mathrm{mmHg})$ & & $189(75.9)$ \\
$>180 / 100$ & $68(79.1)$ & $60(24.1)$ \\
$<180 / 100$ & $18(20.9)$ & $111(44.6)$ \\
Cerebral hemorrhage $(\mathrm{ml})$ & & $138(55.4)$ \\
$>30$ & $53(61.6)$ & 0.697 \\
$<30$ & $33(38.4)$ & $16(6.4)$ \\
Location & & $36(14.4)$ \\
Brainstem & $7(8.1)$ & $37(14.9)$ \\
Cerebellum & $11(12.8)$ & $108(43.4)$ \\
Lobe & $10(11.6)$ & $52(20.9)$ \\
Basal ganglia & $38(44.2)$ & $10.69 \pm 3.67$ \\
Thalamus & $20(23.3)$ & $30(12.0)$ \\
GCS score & $6.13 \pm 2.68$ & 0.006 \\
Hematosepsis & $21(24.4)$ & 0.183 \\
\hline A & &
\end{tabular}

Age and GCS score was expressed as mean \pm standard deviation. Sex, blood pressure, cerebral hemorrhage, location and hematosepsis were expressed as n (\%). GCS, Glasgow Coma Scale.

Table II. Univariate analysis of risk factors of gastrointestinal hemorrhage in patients with acute cerebral hemorrhage.

\begin{tabular}{|c|c|c|c|}
\hline Factor & HR & $95 \% \mathrm{CI}$ & P-value \\
\hline \multicolumn{4}{|l|}{ Sex } \\
\hline Male & 1 & Reference & \\
\hline Female & 1.13 & $0.91-1.29$ & 0.526 \\
\hline \multicolumn{4}{|l|}{ Age (years) } \\
\hline$<60$ & 1 & Reference & \\
\hline$>60$ & 1.18 & $0.87-1.32$ & 0.379 \\
\hline \multicolumn{4}{|c|}{ Blood pressure (mmHg) } \\
\hline$<180 / 100$ & 1 & Reference & \\
\hline$>180 / 100$ & 1.33 & $0.92-1.47$ & 0.023 \\
\hline \multicolumn{4}{|c|}{ Cerebral hemorrhage (ml) } \\
\hline$<30$ & 1 & Reference & \\
\hline$>30$ & 1.38 & $1.26-1.49$ & 0.004 \\
\hline \multicolumn{4}{|l|}{ Location } \\
\hline Brainstem & 1 & Reference & \\
\hline Cerebellum & 0.85 & $0.62-1.23$ & 0.042 \\
\hline Lobe & 0.76 & $0.56-1.25$ & 0.031 \\
\hline Basal ganglia & 0.89 & $0.65-1.08$ & 0.035 \\
\hline Thalamus & 0.82 & $0.64-1.25$ & 0.039 \\
\hline \multicolumn{4}{|l|}{ GCS score } \\
\hline$>8$ & 1 & Reference & \\
\hline$<8$ & 1.43 & $1.28-1.67$ & 0.017 \\
\hline \multicolumn{4}{|l|}{ Septicemia } \\
\hline No & 1 & Reference & \\
\hline Yes & 2.87 & $2.52-3.06$ & $<0.001$ \\
\hline
\end{tabular}

GCS, Glasgow Coma Scale; HR, hazard rate; CI, confidence interval. gastrointestinal hemorrhage in patients with cerebral hemorrhage $(\mathrm{P}<0.05$; Table III).

Prognostic analysis. Among the 86 patients with acute cerebral hemorrhage combined with gastrointestinal hemorrhage, 32 patients died, resulting in a mortality rate of $37.20 \%$. In the negative control group without gastrointestinal hemorrhage, 34 mortalities occurred among the 249 cerebral hemorrhage patients, and the mortality rate in this group was $13.65 \%$. A significant difference in mortality was identified between the two groups $(\mathrm{P}<0.05$; Fig. 3$)$.

\section{Discussion}

Immune inflammatory response after cerebral hemorrhage is a complex pathophysiological process, and cerebral edema and inflammatory reactions are crucial factors in promoting the disease condition of cerebral hemorrhage. Inflammatory reaction was previously considered to be the result of a stress response. It has been reported that the hyperacute inflammatory reaction in response to cerebral hemorrhage exerts a protective effect on promoting hemostasis and reducing hematoma expansion (11). A series of pathophysiological processes have been indicated to be involved in cerebral hemorrhage. Inflammation around the hematoma has an important influence on the degree of neurological impairment, which manifests as aggregation of neutrophils, macrophages or monocytes, and activation of microglia (12).

Gastrointestinal hemorrhage is a serious complication of acute stroke and traumatic brain injury. Peptic ulcers resulting from intracranial lesions are called Cushing ulcers (13). With the improvement of clinical diagnosis and treatment, 
A

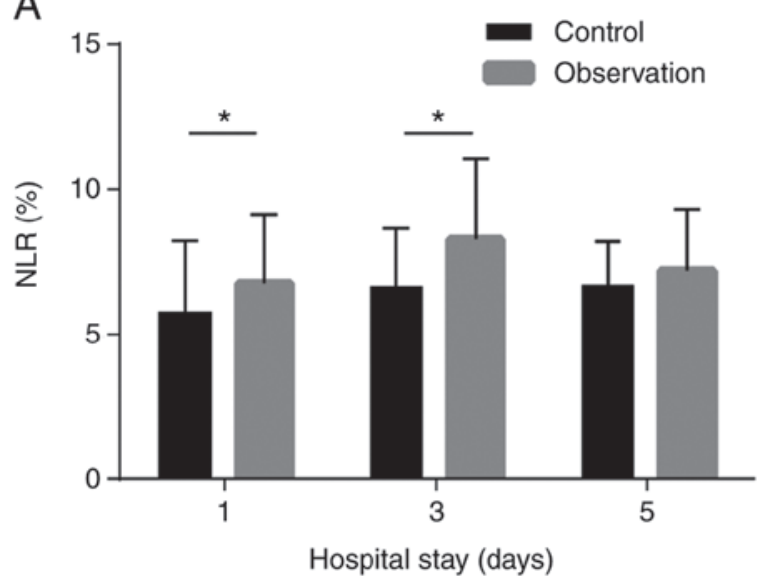

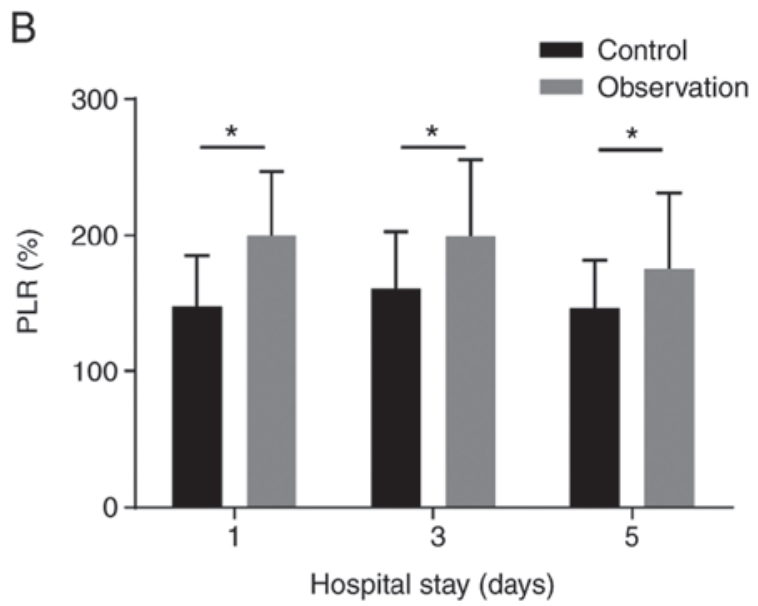

Figure 1. Comparisons of NLR and PLR. (A) The NLR in the observation group was significantly higher than that in the negative control group on the 1st and 3rd day after admission. (B) The NLR in the observation group was significantly higher than that in the negative control group on the 1st, 3rd and 5th day after admission. " $\mathrm{P}<0.05$. NLR, neutrophil to lymphocyte ratio; PLR, platelet to lymphocyte ratio.

A

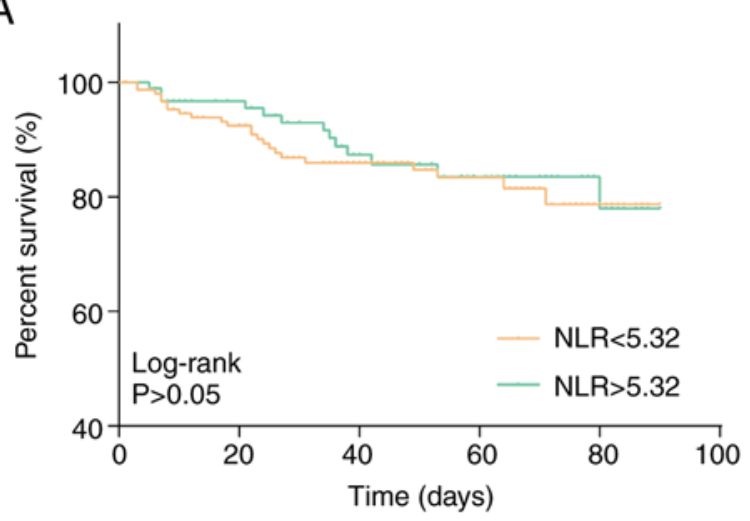

C

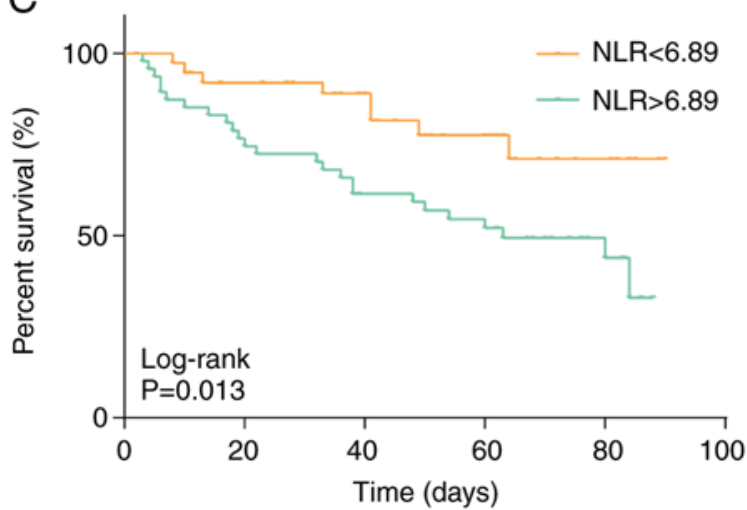

B
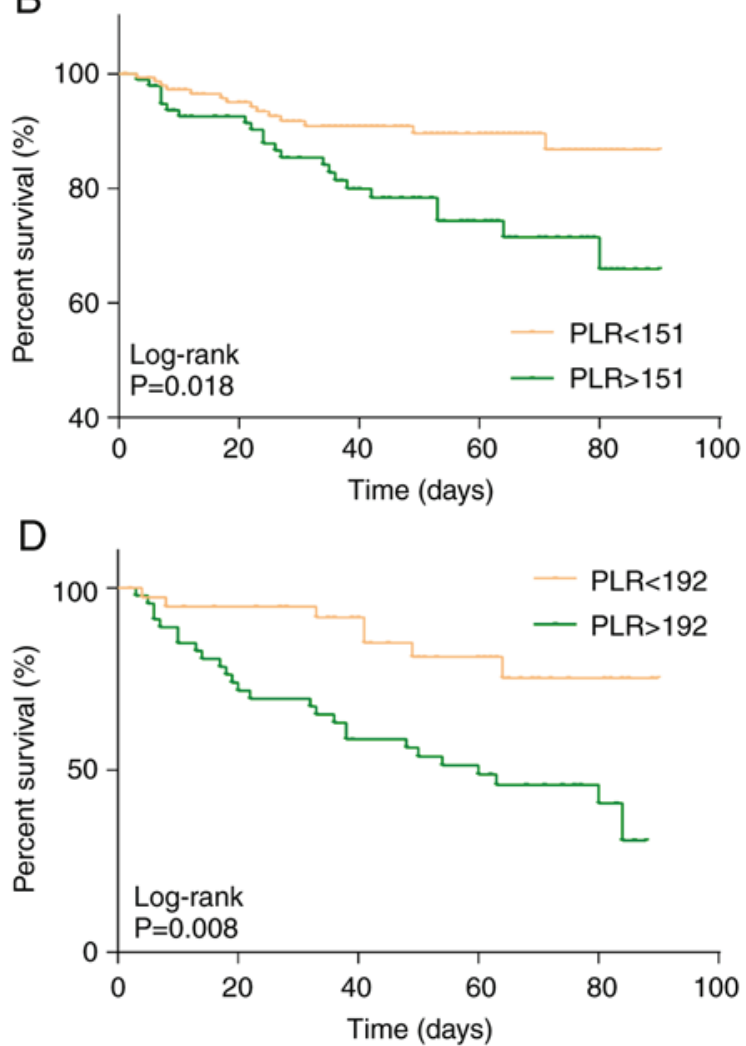

Figure 2. Association between NLR, PLR and overall survival. (A) There was no significant difference between NLR and overall survival in the negative control group. (B) The PLR was negatively associated with overall survival in the negative control group. (C) The NLR was negatively associated with overall survival in the observation group. (D) The PLR was negatively associated with overall survival in the observation group. NLR, neutrophil to lymphocyte ratio; PLR, platelet to lymphocyte ratio.

the incidence of gastrointestinal hemorrhage has markedly decreased $(14,15)$. Wijdicks et al (15) reported that the incidence of stroke complicated with gastrointestinal hemorrhage was $0.80 \%$ in stroke patients. Chen et al (16) determined that $20.5 \%$ of elderly patients with acute stroke experienced gastrointestinal hemorrhage as a complication.

Previous studies on the prognosis of acute spontaneous cerebral hemorrhage mainly focused on the association between the leukocyte count and inflammatory reaction (17). Elevation of WBC in the acute phase of cerebral hemorrhage has been indicated to be positively associated with neurological impairment and 3-month mortality. However, the number of WBC is susceptible to interference from various factors such as diets, drugs and the endocrine system. Imtiaz et al (18) demonstrated that lymphocytes may protect endothelial cells and alleviating the inflammatory response. Imbalance of 
Table III. Multivariate logistic regression analysis of risk factors of gastrointestinal hemorrhage in patients with acute cerebral hemorrhage.

\begin{tabular}{lccrrr}
\hline Factor & B & SE & Wald & OR & 95\% CI \\
\hline Cerebral hemorrhage $>30 \mathrm{ml}$ & 0.879 & 0.336 & 7.235 & 1.268 & $1.004-1.210$ \\
GCS score $<8$ & 1.874 & 0.635 & 11.736 & 1.392 & $0.120-2.102$ \\
Hematosepsis & 1.774 & 0.235 & 13.645 & 3.125 & $1.268-7.495$ \\
\hline
\end{tabular}

GCS, Glasgow Coma Scale; B, regression coefficient; SE, standard error; OR, odds ratio; CI, confidence interval.

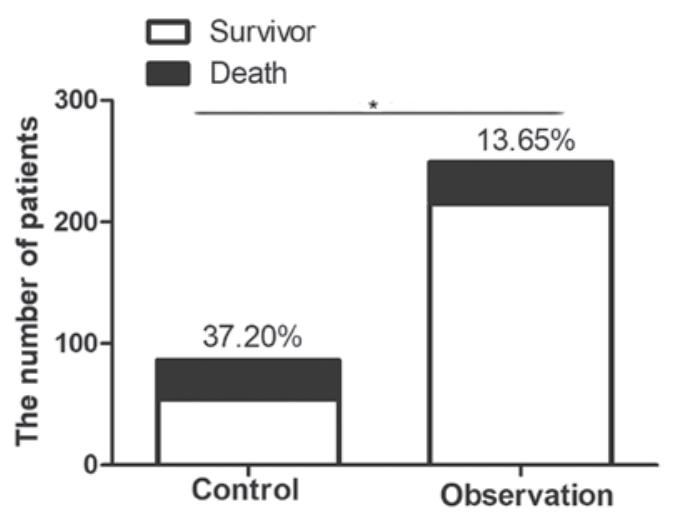

Figure 3. Comparison of mortality between the two groups. ${ }^{*} \mathrm{P}<0.05$.

neutrophils and lymphocytes exaggerates the inflammatory response. The NLR is an indicator of the inflammatory state of the body, with a higher NLR indicating a more severe inflammatory response. Utilization of the NLR as an indicator of inflammation has numerous advantages, including easy accessibility and low cost. Gokhan et al (19) reported that the NEU and NLR in patients with acute cerebral hemorrhage were higher than those in controls. They also suggested that NLR was significant for evaluating the severity of acute cerebral hemorrhage, which was consistent with the study by Lattanzi et al (20). The NLR and PLR have a higher sensitivity and reliability for prognosis in patients with cerebral hemorrhage compared with those of NEU and WBC.

Through these in-depth studies, the association between PLR and the prognostic significance of cerebrovascular disease has been recognized. Altintas et al (21) analyzed 57 patients who were treated by endovascular therapy after acute ischemic stroke. Their results indicated that the PLR was able to reflect an in vivo inflammatory state prior to thrombosis. Elevation of PLR was associated with poor vascular recanalization after treatment with endovascular therapy and poor prognosis over 3 months. Tao et al (22) analyzed the pre-operative routine blood test results of 247 patients with subarachnoid hemorrhage and revealed that an elevated PLR was associated with neurological impairment within 90 days after the onset of subarachnoid hemorrhage.

Based on the previous studies, it may be speculated that the NLR and PLR are associated with cerebral hemorrhage combined with gastrointestinal hemorrhage. In the present study, patients were assigned to an observation group and a negative control group depending on whether they experienced gastrointestinal hemorrhage after cerebral hemorrhage. It was revealed that the NLR and PLR in the observation group were higher than those in the control group. The occurrence time of gastrointestinal hemorrhage is generally 1-2 weeks after acute cerebral hemorrhage (16). Similarly, the occurrence time of gastrointestinal hemorrhage in the observation group of the present study was 1-2 weeks after cerebral hemorrhage. Since the blood was collected on the 1st, 3rd and 5th days of hospitalization, it may be speculated that the elevation of NLR and PLR is involved in the occurrence of gastrointestinal hemorrhage. The NLR and PLR in the observation group were negatively associated with overall survival, whereas only the PLR in the negative control group was identified to be negatively associated with overall survival. The present results were similar to those of previous studies $(19,20,22)$. Future studies by our group, we will aim at further investigating the association between the NLR and PLR and mortality in the two groups. Furthermore, logistic regression analysis indicated that cerebral hemorrhage $>30 \mathrm{ml}$, lower GCS score and hematosepsis were independent risk factors of gastrointestinal hemorrhage in patients with acute cerebral hemorrhage. Therefore, gastrointestinal hemorrhage should be closely monitored in patients with cerebral hemorrhage, particularly in those with the above risk factors. The present results provide further information for the prediction of gastrointestinal hemorrhage after acute cerebral hemorrhage, which may have important significance for the timely diagnosis and treatment of cerebral hemorrhage combined with gastrointestinal hemorrhage. However, the present study was retrospective and the sample size was relatively small. In the future, more samples should be included and multicenter randomized controlled trials should be performed to validate the conclusions of the present study.

In conclusion, elevations of the NLR and PLR were associated with gastrointestinal hemorrhage in patients with acute cerebral hemorrhage. A higher NLR and PLR were negatively associated with overall survival and prognosis. Furthermore, cerebral hemorrhage $>30 \mathrm{ml}$, lower GCS score and hematosepsis were independent risk factors of gastrointestinal hemorrhage in patients with acute cerebral hemorrhage.

\section{Acknowledgements}

Not applicable.

\section{Funding}

The current study was supported by Suzhou Science and Education Xingwei Youth Science and Technology Project (grant no. KJXW2017075), Nantong University-level Natural 
Science Research Fund Project (grant no. 17ZYZ34) and Wujiang First People's Hospital Research Fund Project (grant nos. hospital201708 and hospital201907).

\section{Availability of data and materials}

All data generated or analyzed during the present study are included in this published article.

\section{Authors' contributions}

YZo and YZh designed the study and performed the experiments. YZo, WZ and $\mathrm{CH}$ collected the data, WZ and $\mathrm{CH}$ analyzed the data, and $\mathrm{YZo}$ and $\mathrm{YZh}$ prepared the manuscript. All authors read and approved the final manuscript.

\section{Ethics approval and consent to participate}

This study was approved by the ethics committee of the First People's Hospital of Wujiang District (Suzhou, China). Written informed consent was obtained from all participants prior to the study.

\section{Patient consent for publication}

The patients or their guardians provided written informed consent for publication.

\section{Competing interests}

The authors declare that they have no competing interests.

\section{References}

1. Santoni M, Andrikou K, Sotte V, Bittoni A, Lanese A, Pellei C, Piva F, Conti A, Nabissi M, Santoni G and Cascinu S: Toll like receptors and pancreatic diseases: From a pathogenetic mechanism to a therapeutic target. Cancer Treat Rev 41: 569-576, 2015

2. O'Neill LA: The interleukin-1 receptor/Toll-like receptor superfamily: 10 years of progress. Immunol Rev 226: 10-18, 2008.

3. Kawai T and Akira S: The role of pattern-recognition receptors in innate immunity: Update on toll-like receptors. Nat Immunol 11: 373-384, 2010

4. Kong Y and Le Y: Toll-like receptors in inflammation of the central nervous system. Int Immunopharmacol 11: 1407-1414 2011.

5. Abdi J, Engels F, Garssen J and Redegeld F: The role of toll-like receptor mediated signalling in the pathogenesis of multiple myeloma. Crit Rev Oncol Hematol 80: 225-240, 2011.
6. Hammond MD, Taylor RA, Mullen MT, Ai Y, Aguila HL, Mack M, Kasner SE, McCullough LD and Sansing LH: CCR2 ${ }^{+}$ Ly6C(hi) inflammatory monocyte recruitment exacerbates acute disability following intracerebral hemorrhage. J Neurosci 34: 3901-3909, 2014.

7. Wang $\mathbf{J}$ and Dore $\mathbf{S}$ : Inflammation after intracerebral hemorrhage. J Cereb Blood Flow Metab 27: 894-908, 2007.

8. Atkinson JJ and Senior RM: Matrix metalloproteinase-9 in lung remodeling. Am J Respir Cell Mol Biol 28: 12-24, 2003.

9. Tian XC, Liu XL, Zeng FR, Chen $Z$ and Wu DH: Platelet-to-lymphocyte ratio acts as an independent risk factor for patients with hepatitis B virus-related hepatocellular carcinoma who received transarterial chemoembolization. Eur Rev Med Pharmacol Sci 20: 2302-2309, 2016.

10. Suppiah A, Malde D, Arab T, Hamed M, Allgar V, Smith AM and Morris-Stiff G: The prognostic value of the neutrophil-lymphocyte ratio (NLR) in acute pancreatitis: Identification of an optimal NLR. J Gastrointest Surg 17: 675-681. 2013.

11. Morotti A, Phuah CL, Anderson CD, Jessel MJ, Schwab K, Ayres AM,Pezzini A, Padovani A, Gurol ME, Viswanathan A, et al: Leukocyte count and intracerebral hemorrhage expansion. Stroke 47: 1473-1478, 2016.

12. Gong C, Hoff JT and Keep RF: Acute inflammatory reaction following experimental intracerebral hemorrhage in rat. Brain Res 871: 57-65, 2000.

13. Wijdicks EF: Cushing's ulcer: The eponym and his own. Neurosurgery 68: 1695-1698, 2011.

14. Crooks CJ, Card TR and West J: Excess long-term mortality following non-variceal upper gastrointestinal bleeding: A population-based cohort study. PLoS Med 10: e1001437, 2013.

15. Wijdicks EF, Fulgham JR and Batts KP: Gastrointestinal bleeding in stroke. Stroke 25: 2146-2148, 1994.

16. Chen CM, Hsu HC, Chuang YW, Chang $\mathrm{CH}$, Lin $\mathrm{CH}$ and Hong CZ: Study on factors affecting the occurrence of upper gastrointestinal bleeding in elderly acute stroke patients undergoing rehabilitation. J Nutr Health Aging 15: 632-636, 2011.

17. Agnihotri S, Czap A, Staff I, Fortunato G and McCullough LD: Peripheral leukocyte counts and outcomes after intracerebral hemorrhage. J Neuroinflammation 8: 160, 2011.

18. Imtiaz F, Shafique K, Mirza SS, Ayoob Z, Vart P and Rao S: Neutrophil lymphocyte ratio as a measure of systemic inflammation in prevalent chronic diseases in asian population. Int Arch Med 5: 2, 2012.

19. Gokhan S, Ozhasenekler A, Mansur DH, Akil E, Ustundag M and Orak M: Neutrophil lymphocyte ratios in stroke subtypes and transient ischemic attack. Eur Rev Med Pharmacol Sci 17: 653-657, 2013.

20. Lattanzi S, Cagnetti C, Provinciali L and Silvestrini M: Neutrophil-to-lymphocyte ratio predicts the outcome of acute intracerebral hemorrhage. Stroke 47: 1654-1657, 2016.

21. Altintas O, Altintas MO, Tasal A, Kucukdagli OT and Asil T: The relationship of platelet-to-lymphocyte ratio with clinical outcome and final infarct core in acute ischemic stroke patients who have undergone endovascular therapy. Neurol Res 38: 759-765, 2016.

22. Tao C, Wang J, Hu X, Ma J, Li H and You C: Clinical value of neutrophil to lymphocyte and platelet to lymphocyte ratio after aneurysmal subarachnoid hemorrhage. Neurocrit Care 26: 393-401, 2017. 